\title{
PROTOTIPO DE TECNOLOGÍA EN ASISTENCIA PARA LA ENSEÑANZA DEL BRAILLE
}

\section{PROTOTIPO DE TECNOLOGIA EN ASISTENCIA DE ENSEÑANZA DEL BRAILE}

\begin{abstract}
With the design and manufacture of the device called the ElectronicMechanical System for learning the Braille Literacy (SEMLEB), joint to the application of a tutor-supported methodology, we sought to identify and analyze the factors that facilitate the Braille literacy learning process for blind children in school age and their impact on the Castilian's learning process; to identify letters of the alphabet, phonemes, syllables and some short length words and phrases.
\end{abstract}

Keywords: Braille education; literacy instruction; microcontroller; vocoder

\section{RESUMEN}

Con el diseño y fabricación del dispositivo denominado sistema electrónico mecánico para el aprendizaje la lectoescritura del Braille (SEMLEB), junto con la aplicación de una metodología basada en acompañamiento de un tutor, se buscó determinar y analizar los factores que facilitaran el proceso de aprendizaje de la lectoescritura del sistema Braille para niños ciegos en etapa escolar y sus repercusiones en el aprendizaje del castellano para identificar las letras del alfabeto, los fonemas, las sílabas, y algunas palabras y frases de corta longitud.

Palabras clave: Tecnología en asistencia; didáctica del Braille; enseñanza de la lectoescritura; microcontrolador; codificador de voz

\section{Luis A. Jiménez Hernández}

Ing. Electrónico, Especialista en Teleinformática, MSc. en Educación, docente de la Universidad Manuela Beltrán. Bogotá, Colombia. alexanderjimenez77@yahoo.com

\section{Cesar Augusto Hernandez Suarez} Ing. Electrónico, Especialista en Redes, MSc. en Ciencias de la Información y las Comunicaciones, candidato a Doctor en Ingeniería de Sistemas y Computación. Docente de la Universidad Distrital Francisco José de caldas. Bogotá, Colombia. cahernandezs@udistrital.edu.co

Tipo: Artículo de investigación

Fecha de Recepción: Julio 30 de 2011 Fecha de Aceptación: Septiembre 28 de 2011 


\section{INTRODUCCIÓN}

El Ministerio de Educación Nacional (MEN) en Colombia formuló los estándares básicos de competencias del lenguaje como guía de formación para la educación básica y media, en función de "la importancia que tiene el desarrollo del lenguaje para la formación del individuo y la constitución de la sociedad" [1]. Sin embargo, las dificultades inherentes a la situación de discapacidad pueden llegar a obstaculizar estos objetivos lingüísticos tanto individuales como sociales planteados por el MEN, situación ante la cual es posible apelar a diferentes entidades estatales según el principio constitucional que protege y vela por los derechos fundamentales de los niños colombianos acerca de las condiciones para la educación y la integración social [2]. Dichas dificultades pueden asociarse a la discriminación y exclusión social, las cuales han sido atendidas a solicitud de las comunidades con necesidades especiales, principalmente en las dos últimas décadas, permitiéndoles una mayor participación ciudadana como sujetos portadores de derechos [3].

El reconocimiento de las particularidades individuales surge como principio constitucional de libertades y oportunidades para las personas con diferencias, en este caso, en situación de discapacidad. En el sector educativo se contempla específicamente en la Ley 115 de 1994, señalado en el capítulo I del título III, en donde aparece como "parte integrante del servicio público educativo" [4], y se reglamenta mediante el decreto 2082 de 1996, fundamentado en los siguientes principios: integración social y educativa, desarrollo humano, oportunidad y equilibrio, y soporte específico (hace alusión al tipo de atención específica) [5]. Como resultados de dichas consideraciones se encuentran programas de beneficio social y reinversión de recursos públicos tales como la dotación de kits escolares acordes a las necesidades especiales individuales, ayuda otorgada por la Secretaría de educación del Distrito Capital a 8000 niños sordos o ciegos en el año 2010 [6]. En el servicio público educativo para estudiantes con limitación visual, el Estado cuenta con atención en 421 centros educativos regulares, 71 de ellos con cobertura en el Distrito Capital [7].

En el sector educativo privado también se encuentran Instituciones dedicadas exclusivamente para la atención de las necesidades particulares de aprendizaje de estudiantes en diversas situaciones de discapacidad. Entre los más reconocidos se pueden citar el Colegio Filadelfia para Sordos (propiedad de la Asociación Cristiana Manos en Acción), el cual brinda sus servicios a sordos, sordo-ciegos y sordos con limitaciones [8], y el Instituto para Niños Ciegos Fundación Juan Antonio Pardo Ospina (fundación privada sin ánimo de lucro), el cual brinda educación formal y especial a limitados visuales con o sin retardo mental [9].

Como resultado de un continuo trabajo investigativo, iniciado hace varios años acerca de la situación de la población discapacitada y de cómo aportar soluciones integrales de tipo interdisciplinario, surgió un proyecto para facilitar los procesos de aprendizaje del lenguaje para niños ciegos. La Universidad Manuela Beltrán inició una propuesta investigativa desarrollada por el grupo de investigación en Aplicabilidad Tecnológica, adscrito a la Gerencia I\&D (Investigación y Desarrollo), trabajo orientado a la invención de una tecnología de apoyo, consistente en la construcción de una herramienta tecnológica y didáctica denominada SEMLEB o Sistema electrónico mecánico para el aprendizaje de la lectoescritura del Braille, contando también con una metodología apropiada para orientar el uso y la interacción con el dispositivo. Los lineamientos para su diseño se basaron en los requisitos que deben reunir las ayudas técnicas, tales como:

- Responder a las necesidades para las que han sido concebidas.

- Seguras, evitando riesgos innecesarios.

- Sencillas, manejo sencillo para poder usarlas solos.

- Fabricadas con materiales resistentes, duraderos, de fácil limpieza, estéticos y de bajo costo [10].

Según la Academia Nacional de Ciencias del Consejo Nacional de Investigaciones de los Es- 
tados Unidos, resulta difícil reconocer las palabras escritas cuando no se tiene la noción de su representación auditiva sistemática [11]. Por este motivo se consideró como objetivo del proyecto la necesidad de asociar las letras del sistema Braille a sus correspondientes fonemas, incluyendo funciones para la composición y reproducción de sonidos de letras, fonemas, sílabas, palabras y frases empleados en las cartillas de aprendizaje del lenguaje castellano escrito.

\section{FINANCIACIÓN}

Este proyecto contó con la financiación de la Universidad Manuela Beltrán, del Departamento Administrativo de Ciencia, Tecnología e Innovación COLCIENCIAS (al ser presentado como propuesta ante la Subdirección de Programas de Innovación y Desarrollo Empresarial) y de la entidad estatal Servicio Nacional de Aprendizaje SENA. Como principal antecedente se contó con un importante trabajo orientado a la iniciación del niño ciego en el aprendizaje del Braille, del cual surgieron los principales lineamientos para la planeación y estructuración del proyecto SEMLEB. Dicho trabajo denominado Sistema de Lectoescritura en Braille (SLE) también tuvo el auspicio de las instituciones mencionadas. Se espera continuar con la colaboración logística y de equipo humano de diferentes instituciones públicas y privadas para dar continuidad al proceso investigativo iniciado, puesto que los alcances de nivel formativo y de desarrollo de los niños ciegos son de alto impacto en la comunidad discapacitada.

\section{METODOLOGÍA}

El sistema Braille está conformado por un conjunto de puntos organizados en una matriz, llamado también "signo generador" cuyas combinaciones representan letras, números, signos de puntuación y otros caracteres especiales. Dicha organización se encuentra de forma tradicional en un arreglo de tres filas y dos columnas, permitiendo hasta un total de 64 combinaciones (incluyendo el espacio entre palabras), resultado obtenido de la operación expresada como $2^{\wedge} 6$ (dos elevado a la potencia seis), con la cual se pueden abarcar fácilmente las letras del alfabeto y algunos signos adicionales. Con el fin de ampliar la capacidad de representaciones con el signo generador se adicionó al sistema la representación por "símbolos dobles" [12], como en el caso de los dígitos numéricos y las letras mayúsculas. Otros esquemas trabajan con 4 filas, incluyendo una fila más en la parte inferior del signo, con los cual se extiende la cantidad hasta 256 combinaciones, como por ejemplo, para la identificación con el byte o palabra digital, por lo que ha sido denominado como "braille informático" [13]. Para los objetivos del proyecto, dada su condición de ser una tecnología empleada como herramienta didáctica para el aprendizaje de la lectoescritura en edad escolar, solamente fue necesario el uso del código de seis puntos.

La población a la cual estuvo dirigido el objetivo del proyecto fue la comunidad de limitados visuales, específicamente niños ciegos que estuvieran en etapa preescolar que no presentaran otro tipo de limitación significativa que les impidiera interactuar físicamente con el aparato y escuchar los sonidos que reproduce. Cabe aclarar que la etapa preescolar de cada niño no se determinó por su edad cronológica, sino por el momento de iniciación en el proceso de aprendizaje de la lectoescritura del sistema Braille, en el rango entre ocho y doce años de edad, debido a que no todos los infantes tienen la misma capacidad de comenzar sus estudios básicos debido a las dificultades inherentes a su condición como personas discapacitadas y a la situación económica de sus familias, a pesar de las posibilidades que ofrecen los centros educativos públicos.

El sistema cuenta con una biblioteca de palabras y sonidos almacenados de forma digital, de manera que el componente electrónico encargado del procesamiento de información encuentra los datos correspondientes para la reproducción de audio y dar al usuario una realimentación del proceso. Las palabras fueron seleccionadas a partir de varios textos educativos que se emplean para el desarrollo de competencias lingüísticas en los primeros grados escolares. Para determinar el tipo de so- 
nidos de voz que debía reproducir el sistema, se realizó un estudio acerca de la facilidad de comprensión por parte de un determinado grupo de niños voluntarios, del cual se obtuvo lo siguiente: a) la voz del sintetizador electrónico carece de una entonación adecuada para la enseñanza del castellano, por lo cual se prefirió grabar voz humana; b) realizando una comparación de género, los niños prefirieron una voz masculina, argumentando que se entendía de forma más clara que la voz femenina. Para recopilar los sonidos de las palabras se contó con la colaboración de un locutor profesional y se emplearon los estudios de grabación propios de la Universidad Manuela Beltrán.

Para el desarrollo tecnológico se contó con un grupo conformado por Ingenieros Electrónicos $\mathrm{y}$ un Diseñador Industrial, pertenecientes al Grupo de Investigación en Aplicabilidad Tecnológica, cada uno encargado del diseño, montaje y evaluación técnica de los diferentes módulos funcionales del dispositivo conformado por partes electrónicas y mecánicas, contando también con la participación de estudiantes de ingeniería electrónica y biomédica. El desarrollo del método de aplicación y validación del aparato estuvo a cargo de un competente grupo de especialistas conformado por dos Tiflólogos (uno de ellos con discapacidad visual, cuyos aportes permitieron obtener apreciaciones y valoraciones más objetivas acerca del dispositivo y las pruebas técnicas), un Médico, un Fonoaudiólogo y un Fisioterapeuta, con el apoyo de los Docentes de las entidades educativas en donde se realizaron las pruebas piloto.

En las pruebas de ambiente controlado se tuvo la participación de Estudiantes, Profesionales y Personas Adultas ciegas, con las cuales se recopiló información para realizar ajustes tanto del dispositivo como del método de aplicación. Finalmente se realizaron pruebas reales con población invidente en dos Colegios Distritales: niños conocedores del Braille y niños de grado cero, quienes iniciaron su proceso de aprendizaje del Braille.

Finalmente se llegó al desarrollo de un sistema tecnológico y didáctico como se muestra en la fig. 1, basado en el uso de un dispositivo tecnológico en el cual participa un docente de apoyo, permitiendo al niño con limitación visual realizar un ejercicio de escritura Braille en el módulo mecánico, el cual es procesado por el módulo electrónico emitiendo una respuesta audible que sirve como realimentación en el proceso de aprendizaje, todo esto empleando un método de enseñanza y seguimiento para conducir al niño al desarrollo de las competencias lingüísticas básicas para leer y escribir en castellano.

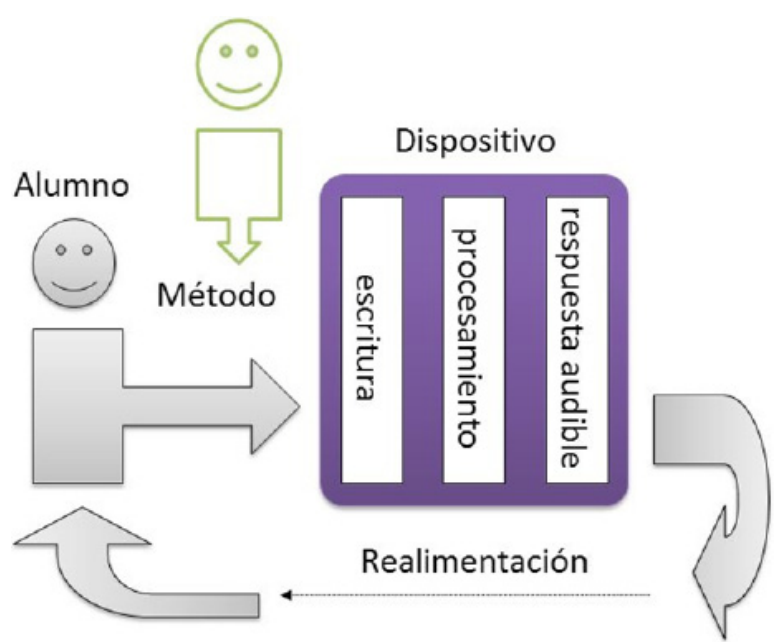

Fig. 1. Interacción entre el niño y los componentes del sistema

Con base en los resultados obtenidos en las diferentes etapas del proceso investigativo, se determinaron aspectos importantes acerca del diseño físico del sistema, en el cual se incluyeron aspectos tales como: a) botones que desempeñen funciones de manera sencilla para cualquier usuario; b) formas geométricas que estimulen otras habilidades en el niño; c) uso de colores que brinden una apariencia lúdica al dispositivo, principalmente como factor psicológico, para las demás personas que tengan contacto con el aparato; d) diferentes tamaños de cajetín, hasta ajustarse al tamaño real del signo generador, para permitir al niño trabajar en diferentes grados de motricidad; e) mantener el concepto de reversibilidad de la lectoescritura del sistema Braille, y facilitar su dominio; f) un sistema modular que permita el intercambio de partes móviles según el nivel de desempeño del niño; g) un ajuste de volumen 
e incluso la posibilidad de conectar audífonos personales; entre otras consideraciones de uso práctico.

\section{RESULTADOS}

En la planeación del desarrollo tecnológico se determinó que, según las funciones que debía cumplir cada uno de los elementos que componían el dispositivo, lo más conveniente era trabajar de forma modular para que cada parte del sistema electrónico, mecánico y ergonómico pudiese construirse de manera óptima y su elaboración estuviera a cargo de un equipo humano dedicado a cada caso, trabajando en conjunto de forma integrada. Finalmente se establecieron los módulos que se muestran en la Fig. 2 y se explican a continuación en mayor detalle.

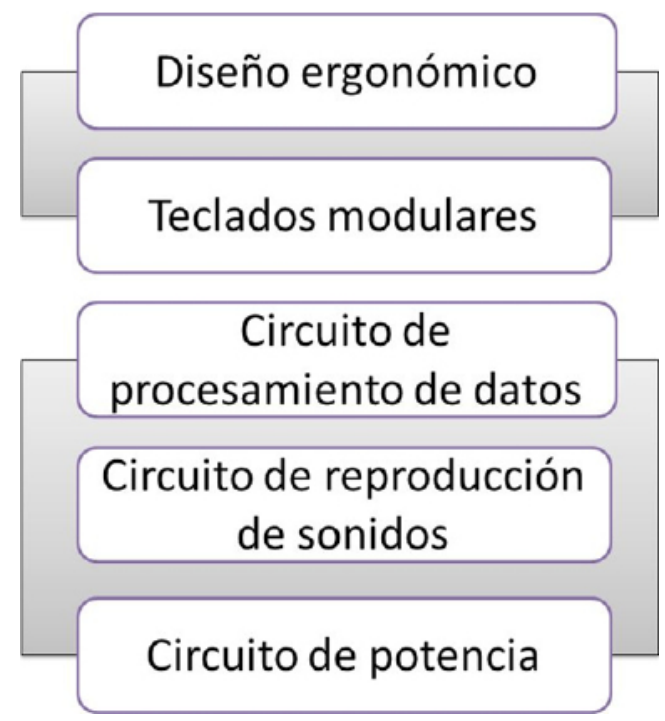

Fig. 2. Módulos que conforman el diseño y desarrollo tecnológico

\subsection{Diseño ergonómico}

Se analizaron los tipos de interacción física y psicológica de las personas con limitación visual y sus objetos de uso cotidiano, didáctico, lúdico y tecnológico, lo cual condujo a diseñar y construir una carcasa con características apropiadas no solamente para la fácil manipulación del dispositivo empleando varios planos de acción, sino también para despertar en el usuario una sensación estética de las proporciones y que resulte agradable al tacto, principalmente por el potencial que tiene este sentido para las personas ciegas.

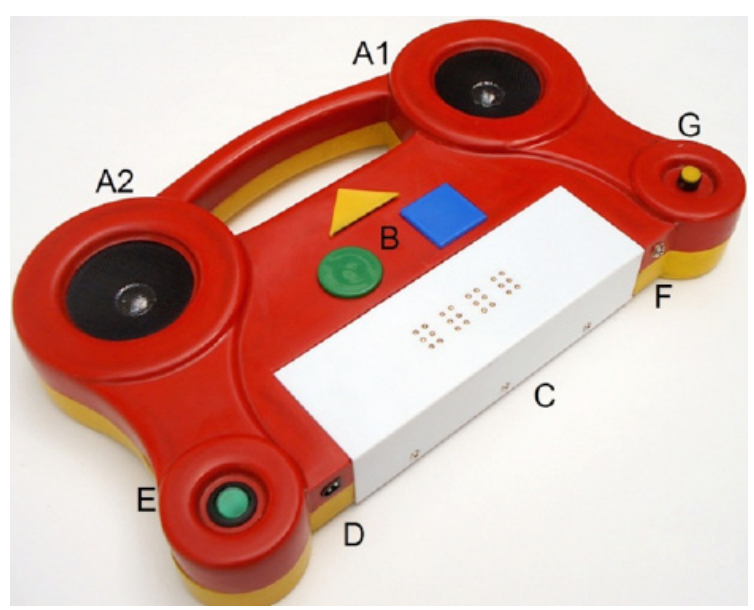

Fig. 3. Vista anterior del dispositivo

El resultado fue un "estudio antropométrico de escalamiento áureo" [14], el cual condujo a un dimensionamiento y una distribución como se muestra en la Fig. 3, en donde aparecen las siguientes partes funcionales: a) ubicación bilateral de los parlantes inductivos a través de los cuales se emiten los sonidos del dispositivo, cubiertos con protectores contra los golpes para proteger los conos; b) ubicación central de los botones de reproducción, cada uno con una forma geométrica simple que los niños pueden reconocer y recordar fácilmente, cada uno de ellos asociado a una función diferente; c) ubicación inferior de los tres módulos intercambiables, uno conectado a la vez, para la lectoescritura del Braille con cajetines de varios tamaños y en diferente cantidad; d) conector de alimentación de energía y carga de la batería eléctrica; e) botón de encendido del aparato; f) conector para uso de audífono, en caso de que el usuario quiera interactuar con el dispositivo y escuchar los sonidos individualmente, g) perilla para ajustar el nivel de volumen del sonido. El material empleado en el prototipo fue fibra de vidrio, ofreciendo una alta rigidez y durabilidad permitiendo una manipulación que no requiera extrema delicadeza como en la mayoría de equipos tecnológicos comunes, especialmente si se considera que los usuarios del dispositivo 
son niños en etapa escolar y por lo general son inquietos y poco cuidadosos con las cosas.

\subsection{Teclados modulares}

Este módulo está muy relacionado con el diseño de la carcasa puesto que conforma una parte física externa, al mismo tiempo que un componente esencial del dispositivo porque contiene los cajetines a través de los cuales el usuario puede realizar tanto la escritura, empleando un punzón de manera similar a la escritura del Braille sobre el papel, como la lectura, al invertir el dispositivo y palpando el relieve de cada cajetín en orden, aplicando el principio de reversibilidad de la lectoescritura del Braille.

Cada teclado cuenta internamente con un circuito eléctrico conformado por diminutos interruptores de corriente ubicados en un arreglo matricial. Para su fabricación, primero se puso a prueba el acople mecánico de los interruptores con los bastoncillos que son desplazados por el usuario cada vez que pulsa una posición con el punzón, correspondiendo con el cierre o apertura de un contacto eléctrico. Este proceso corresponde con la emulación de la escritura del Braille sobre papel, cuando el limitado visual punza cada punto del signo generador, permitiéndole adquirir la destreza requerida para memorizar tanto mental (identificación de los signos) como físicamente (memoria muscular) las letras del alfabeto y escribir de forma continua. En la Fig. 4 se muestra un montaje de prueba funcional en donde se corroboró y analizó la funcionalidad práctica de este mecanismo para una línea de ocho cajetines, en donde se puede apreciar las partes mecánicas del sistema: a) bastoncillos para activar los interruptores, b) interruptores eléctricos dispuestos en dos niveles, ubicados en circuitos impresos interconectados mediantes zócalos.

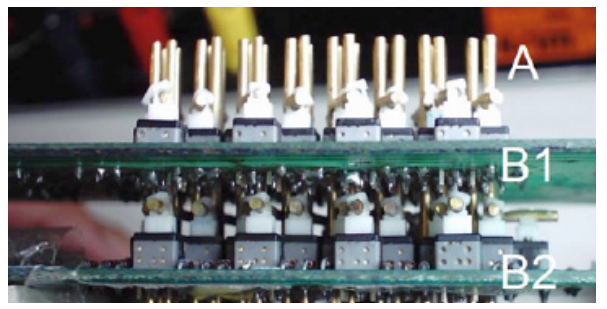

Fig. 4. Vista interna de un teclado de prueba funcional
Para cada teclado se determinó una cantidad de cajetines apropiada según la dificultad y el nivel académico de enseñanza y aprendizaje del idioma castellano, de la siguiente manera: a) el primer teclado constó de un solo cajetín, cuyas medidas corresponden a un signo generador con puntos de $4.8 \mathrm{~mm}$ de diámetro, con espacio de $9 \mathrm{~mm}$ entre ejes; b) el segundo teclado contiene cuatro signos generadores con puntos de $3.2 \mathrm{~mm}$ de diámetro, con espacio de $6 \mathrm{~mm}$ entre ejes; c) el tercer teclado contiene dieciséis signos generadores con las medidas convencionales del Braille escrito. Cuando se enciende el aparato, este reconoce el tipo de teclado dando un mensaje de bienvenida al usuario para recordar el nivel de aprendizaje en el cual se encuentra. Si se tiene insertado el teclado de un cajetín al encender el dispositivo, se escucha el mensaje "vamos a aprender las letras"; con el de cuatro cajetines se escucha "vamos a aprender las sílabas"; con el de dieciséis cajetines se escucha "vamos a aprender palabras completas"; incluso si no hay conectado un teclado al dispositivo, se escucha un mensaje indicando el error al usuario: "por favor, apágame e inserta un teclado".

\subsection{Circuito de procesamiento de datos}

La información proveniente de los teclados y los botones de función, es procesada por un microcontrolador de alta gama del fabricante Freescale Semiconductor, el cual se encarga de realizar las operaciones requeridas para identificar el tipo de teclado, recibir las instrucciones de los botones, codificar los datos de los cajetines y enviar las instrucciones correspondientes al circuito de reproducción de sonidos, así como otras funciones de diagnóstico del sistema electrónico.

En la memoria no volátil del microcontrolador se almacena el programa principal junto con la biblioteca de datos, consistente en las tablas de identidad de letras, sílabas o palabras, y las tablas de direccionamiento de cada vocoder (codificador de voz). La programación fue basada en un algoritmo de identificación y búsqueda de manera que, al capturar el texto del teclado, lo asociara con los datos de la biblioteca de la 
manera más eficiente en términos de tiempo y acceso a la memoria no volátil.

La información de los teclados se halla dispuesta de forma matricial, para lo cual se emplearon puertos lógicos de entrada y salida del microcontrolador, permitiendo realizar una lectura dinámica [15]. Los botones de función se conectaron a puertos de entrada de manera directa, y pulsando cada uno de ellos se desarrolla una tarea diferente, tal como aparece en la tabla 1.

Tabla 1. Funciones de reproducción de los botones

\begin{tabular}{|l|l|l|l|}
\hline Teclado & $\begin{array}{c}\text { Botón } \\
\text { redondo } \\
\text { (letras } \\
\text { individuales) }\end{array}$ & $\begin{array}{c}\text { Botón } \\
\text { cuadrado } \\
\text { (para sílabas) }\end{array}$ & $\begin{array}{c}\text { Botón } \\
\text { triangular } \\
\text { (para } \\
\text { palabras) }\end{array}$ \\
\hline $\begin{array}{l}\text { De } 16 \\
\text { signos }\end{array}$ & Cada letra & $\begin{array}{l}\text { Sílabas de a pa- } \\
\text { labra / sílabas } \\
\text { de las palabras } \\
\text { de la frase }\end{array}$ & $\begin{array}{l}\text { Palabra } \\
\text { completa / frase } \\
\text { completa }\end{array}$ \\
\hline $\begin{array}{l}\text { De } 4 \\
\text { signos }\end{array}$ & Cada letra & Ślaba & $\begin{array}{l}\text { Sílaba / palabra } \\
\text { corta }\end{array}$ \\
\hline $\begin{array}{l}\text { De } 1 \\
\text { signo }\end{array}$ & Letra & Fonema & $\begin{array}{l}\text { Objeto con la } \\
\text { inicial de la letra }\end{array}$ \\
\hline No hay & Deshabilitado & Deshabilitado & Deshabilitado \\
\hline
\end{tabular}

La estructura de datos de la biblioteca permitió organizar y almacenar la información en el microcontrolador, del mismo modo que funciona en un ordenador, lo que permite mejorar el desempeño dinámico y estático [16]. Los datos se organizaron de diferentes maneras: a) en registros individuales, como en las variables; b) en registros dobles, como en las direcciones de acceso a la memoria; c) en cadenas, como las palabras y las sílabas adquiridas desde el teclado; d) en datos encapsulados, los cuales constan de varios datos identificados mediante un prefijo y un sufijo, de longitud y contenido variable según la tarea que se esté ejecutando. Las conexiones hacia el circuito de reproducción de sonidos se realizan mediante puertos de salida (direccionamiento de los vocoders) y de comunicación serial tipo SPI (interfaz periférica serie).

\subsection{Circuito de reproducción de sonidos}

Este módulo almacena y reproduce los sonidos del dispositivo. Una parte consta de dos vocoders del fabricante Winbond Electronics Corporation, en los cuales fueron grabados los sonidos de voz humana como aparece en la tabla 2 .

Tabla 2. Cantidad de sonidos grabados

\begin{tabular}{|l|c|}
\hline \multicolumn{1}{|c|}{ Sonidos almacenados } & Cantidad \\
\hline Letras individuales & 27 \\
\hline Fonemas de las letras & 27 \\
\hline Sílabas o palabras monosílabas & 300 \\
\hline Palabras de ejemplo de cada letra (objetos) & 27 \\
\hline Mensajes del sistema & 6 \\
\hline Letras o palabras de una sola letra & 27 \\
\hline Palabras polisílabas & 763 \\
\hline Total sonidos del equipo & 1177 \\
\hline
\end{tabular}

La otra parte de este módulo consiste en un amplificador de sonido con control de volumen, del fabricante National Semiconductor Corporation, y una conexión para parlantes y audífonos mediante la cual, el usuario puede ajustar las preferencias de realimentación auditiva en el uso del aparato. Tiene una corriente de reposo baja y la potencia de salida para ambos parlantes alcanza hasta $1 \mathrm{~W}$.

\subsection{Circuito de potencia}

Para la alimentación de energía del dispositivo se cuenta con conexión directa a la red eléctrica, mediante el uso de una fuente DC tipo adaptador de corriente, que sirve al mismo tiempo como cargador para la batería interna de $6 \mathrm{~V}$, la cual permite una autonomía de dos horas de uso continuo de lectura-escritura y reproducción de sonidos, tiempo apropiado para una sesión de aprendizaje del niño ciego. El sistema de energía cuenta con dos fuentes reguladas internas, correspondientes a $5 \mathrm{~V}$ y $3 \mathrm{~V}$ para los diferentes circuitos electrónicos.

\section{USO DIDÁCTICO}

La conformación física del aparato permite al usuario emplearlo de manera sencilla y segura, siendo menos requerida la participación del tutor en cuanto a lo técnico, facilitándole su acción docente para dedicarse a las explicaciones 
propias de la enseñanza del idioma castellano, como la sintaxis, la semántica, la ortografía, etc. Después de una introducción breve acerca del funcionamiento del dispositivo, el niño pronto alcanza una apropiación de uso que le permite seguir las actividades indicadas por el maestro. En primer lugar, el niño sostiene el aparato e identifica la cara de lectura (signos Braille en relieve), accediendo a los botones de encendido y de funciones de reproducción de sonidos y escuchando un mensaje de bienvenida según el teclado ubicado en el conector inferior, lo cual le indica al mismo tiempo el nivel de dificultad de la actividad que va a realizar, como aparece en la Fig. 5.

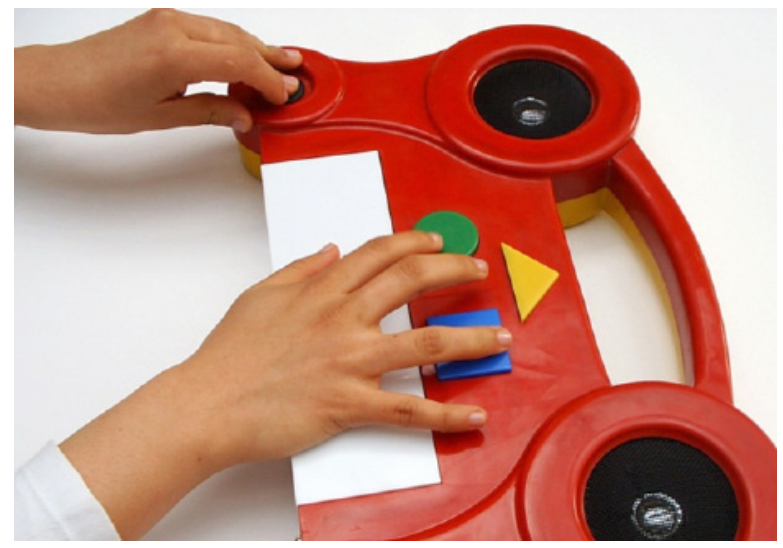

Fig. 5. Identificación de los botones del dispositivo

Para el ejercicio de escribir en Braille, el método consiste en la inversión del aparato, en donde encontrará la cara de escritura y mediante un punzón, el niño acciona los interruptores insertándolo en los orificios de cada signo generador, uno a la vez y de derecha a izquierda como aparece en la Fig. 6, del mismo modo que lo hace con la regleta de cajetines convencional. Una importante ventaja del proceso con el dispositivo es que le permite al niño una iniciación gradual en tamaño de mayor a menor.

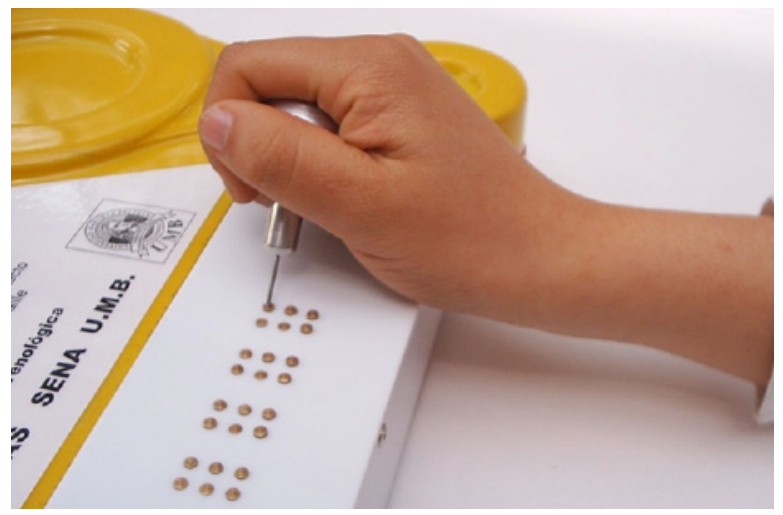

Fig. 6. Uso del punzón en la cara de escritura

Para leer el texto escrito, el niño invierte nuevamente el aparato, dejándolo con la cara de lectura hacia arriba, en donde encuentra en relieve los signos generadores, sobre los cuales puede realizar el ejercicio táctil de identificar cada una de las letras como aparece en la Fig. 7. El niño encuentra también las opciones de reproducción al oprimir los botones con Figuras geométricas, completando el ciclo con la realimentación auditiva, quedando preparado para la siguiente actividad indicada por el tutor.

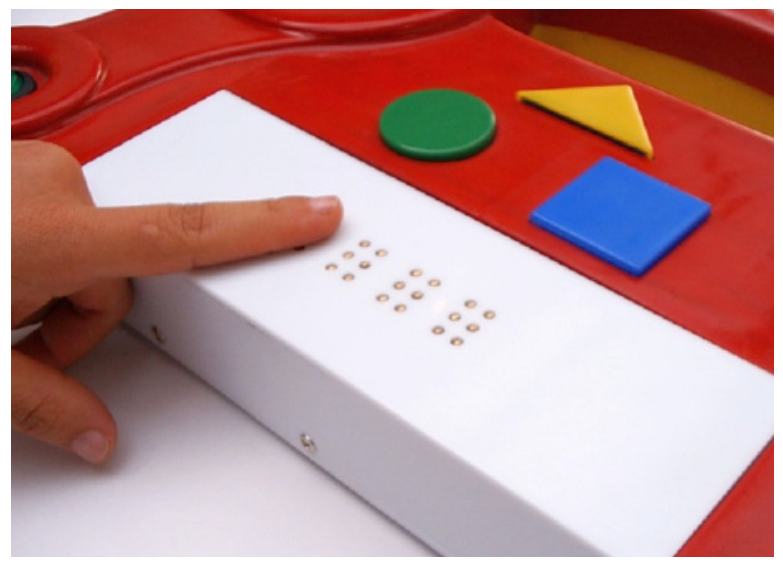

Fig. 7. Lectura táctil en el teclado de cuatro signos generadores

Para avanzar a un nivel superior de enseñanza del Braille, el tutor indica al niño cómo realizar el cambio del teclado, como en el caso de pasar de un ejercicio de lectoescritura de sílabas a otro en donde se empleen palabras completas. La inserción de cada módulo de teclado solo puede hacerse en un sentido, coincidiendo siempre el relieve de los signos generadores 
con la cara de los botones del aparato, como aparece en la Fig. 8.

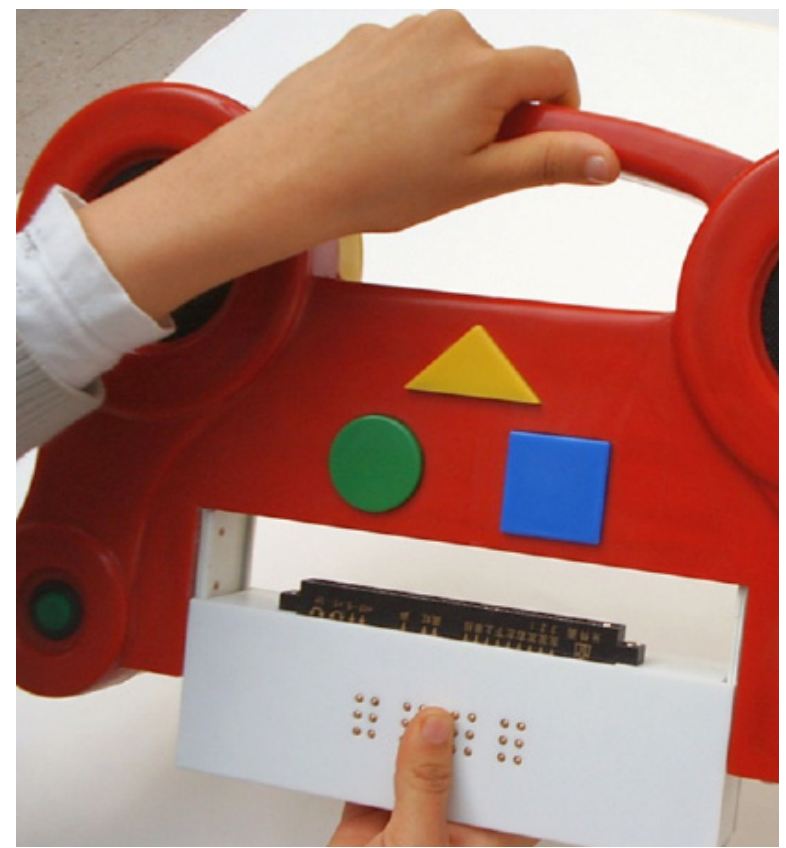

Fig. 8. Inserción de un teclado en el dispositivo

Con poco entrenamiento acerca del uso del aparato, el usuario puede adquirir un completo dominio de su funcionamiento. Según las recomendaciones metodológicas del tutor con respecto al avance y capacidad de trabajo autónomo del niño, puede permitirse el uso independiente del aparato, de manera que el niño pueda seguir realizando actividades individualmente. El dispositivo cuenta con una perilla de ajuste de volumen para cambiarlo al nivel requerido cuando se realiza alguna actividad de aprendizaje individual, e incluso puede emplear audífonos y evitar que el sonido interfiera con las tareas que estén desarrollando otros estudiantes que estén ubicados en un mismo salón de clase, recordando que en los colegios públicos que prestan atención a población con limitación visual en Colombia, los espacios son compartidos simultáneamente con otros niños.

\section{CONCLUSIONES}

Ampliar las posibilidades de aprendizaje de la lectoescritura de los niños con limitación visual a través de herramientas tecnológicas facilita la accesibilidad futura a los campos social, cultural, académico y laboral, mejorando sus condiciones de integración e inclusión y también para adquirir habilidades que le permitan alcanzar una pronta independencia para realizar actividades individuales. El uso tradicional de la regleta basada en la reversibilidad para la escritura con punzón y luego la lectura táctil del relieve en el papel, ha sido el método empleado desde la aparición y difusión del sistema Braille para las personas ciegas. El propio acto de la lectura permite dar significado a los textos de manera similar a la conversación entre seres humanos, por lo cual resulta importante el desarrollo de los sentidos del tacto y de la audición, aprovechando las cualidades de plasticidad del cerebro [17], especialmente para interactuar y relacionarse con el ambiente.

La ayuda didáctica que brinda el dispositivo SEMLEB presenta a los usuarios una alternativa tecnológica y lúdica que puede facilitarles el aprendizaje de la lecto-escritura del Braille, permitiéndoles participar de manera más activa en dicho proceso, al mismo tiempo que una capacidad para adquirir competencias lingüísticas de manera autónoma, permitiéndoles relacionar de manera directa sus acciones de escritura con la composición adecuada de las sílabas y las palabras, de forma inmediata mediante una realimentación auditiva. De este modo, la participación del tutor puede reducirse de manera progresiva, permitiendo al niño con limitación visual explorar la versatilidad del dispositivo y emplearlo como herramienta para descubrir sus potencialidades y así obtener un mejor desenvolvimiento en cuanto a sus procesos cognitivos y meta-cognitivos [18].

Contar con teclados de diferente tamaño de cajetines facilita el aprendizaje del signo generador de manera progresiva, en especial si el niño se encuentra en una etapa temprana de desarrollo de habilidades motoras, del mismo modo que las formas geométricas con las que cuenta la carcasa del aparato permiten una mayor estimulación sensorial que cuando se inicia la enseñanza de la lectoescritura del Braille empleando exclusivamente la regleta, siendo el dispositivo un complemento didáctico muy 
apropiado para despertar el interés de los niños en los procesos educativos del lenguaje escrito.

La característica tecnológica del dispositivo resultó también atractiva para los niños por varias razones: a) se convierte en un instrumento lúdico que motiva el aprendizaje del Braille y del idioma; b) es una herramienta con la que pueden interactuar y experimentar; c) son pocos los aparatos diseñados en su totalidad para personas con discapacidad visual en etapa escolar, de manera que les permite un uso con menor supervisión del tutor, ofreciéndoles mayor utilidad y apropiación como herramienta personal; d) los involucra de una manera más participativa y activa en sus procesos de lectoescritura. Adicionalmente, el diseño modular

Referencias Bibliográficas

[1] M.E.N. Ministerio de Educación Nacional, "estándares básicos de competencias del lenguaje", p. 18, publicación electrónica sin fecha. Consultado en Marzo 17 de 2011, disponible en: http://www.mineducacion. gov.co/1621/articles-116042_archivo_pdf1.pdf

[2] Asamblea Nacional Constituyente, Constitución Política de Colombia, 1991.

[3] Discapacidadcolombia.com, Legislación, publicación electrónica sin fecha. [En linea], consultado en Marzo 17 de 2011, disponible en: http://discapacidadcolombia.com/modules.php ?name $=$ Content $\& p a=$ showpage $\&$ pid $=$ 34

[4] Congreso de Colombia, Ley 115 de 8 de febrero de 1994.

[5] Presidencia de la República, decreto 2082 de 18 de Noviembre de 1996, Colombia.

[6] Secretaría de Educación del Distrito Capital, más de 100 mil estudiantes del Distrito recibirán morrales con útiles escolares, publicación electró- y programable del aparato permite que sea empleado para la enseñanza de otros idiomas que tengan como base el sistema Braille, principalmente si usan alfabetos con caracteres latinos, puesto que las modificaciones serían menores, actualizando las bibliotecas de sonidos y de palabras solamente.

\section{AGRADECIMIENTOS}

Los autores agradecen la inversión económica de las entidades patrocinadoras mencionadas y la valiosa colaboración de las asociaciones colombianas: FENASCOL, INCI y SURCOE, así como a las instituciones educativas públicas distritales de las localidades de Tunjuelito y Fontibón que participaron en el proyecto.

[7] Discapacidadcolombia.com, Centros educativos regulares que atienden población ciega o con baja visión, publicación electrónica sin fecha. [En linea], consultado en Marzo 17 de 2011, disponible en: http://discapacidadcolombia.com/documentos/COLEGIOS INTEGRADORES.pdf

[8] Colegio filadelfia Para Sordos, publicación electrónica sin fecha. [En linea], consultado en Marzo 17 de 2011, disponible en: http://www.filadelfiaparasordos.com/

[9] Instituto para Niños Ciegos Fundación Juan Antonio Pardo Ospina publicación electrónica sin fecha. [En linea], consultado en Marzo 17 de 2011, disponible en: http://institutoparaninosciegos.org/

[10] Entorno accesible M. B. López, y J. J. Ferrero, Ayudas técnicas: concepto, publicación electrónica sin fecha. 
[En linea], consultado en Marzo 17 de 2011, disponible en: http:/www.accesible.es/ayudas_tecnicas_concepto. htm

[11] J. Alegría, M. Carrillo, E. Sánchez. La enseñanza de la lectura, Investigación y ciencia, número 340, Enero de 2005. pp. 8-14.

[12] Discapnet, Fundación ONCE, sistema Braille, publicación electrónica sin fecha. [En linea], consultado en Marzo 17 de 2011, disponible en: http:// usuarios.discapnet.es/ojo_oido/sistema braille.htm

[13] A. García, Año Internacional del Brai1le, Enero 12 de 2009. [En línea]. consultado en Marzo 17 de 2011, disponible en: http://www.consumer.es/web/ es/solidaridad/proyectos_y_campanas/2009/01/12/182555.php

[14] M.A. Dussán, L.A. Jiménez, C.A. Hernández, L.G. Peñaranda, F.A. Villamizar. Sistema electrónico mecáni- co para el aprendizaje de la lecto-escritura del Braille, Umbral Científico, Universidad Manuela Beltrán, Bogotá D.C., Diciembre de 2004, p. 64.

[15] M.A. Dussán, L.A. Jiménez, C.A. Hernández, L.G. Peñaranda, F.A. Villamizar, Manual de referencia SEMLEB, resultado de investigación no publicado, Universidad Manuela Beltrán, 2005.

[16] B. Bierschenk, Data structures for 8-Bit microcontrollers, Motorola semiconductor application note AN1752. Motorola Inc, 1998.

[17] B. Röder, Percepciones sensoriales de los invidentes, mente y cerebro, número 7, año 2004, pp. 26-29,

[18] L.A. Jiménez. La ejecución y el control en la solución de problemas empleando métodos heurísticos y situaciones contextualizadas. Tesis de maestría no publicada, Bogotá, Pontificia Universidad Javeriana, 2009 